\title{
Sparse volumetric PZT array with density tapering
}

\author{
Hendrik J Vos ${ }^{\text {a,b }}$, Enrico Boni ${ }^{\text {c }}$, Alessandro Ramalli ${ }^{\text {c,d }}$, Filippo Piccardi ${ }^{\text {a,c }}$, Andrea Traversi ${ }^{\text {a,c }}$, Davide Galeotti ${ }^{\text {a,c }}$, Emile C \\ Noothout $^{\mathrm{a}}$, Verya Daeichin ${ }^{\mathrm{a}}$, Martin D Verweij ${ }^{\mathrm{a}, \mathrm{b}}$, Piero Tortoli ${ }^{\mathrm{c}}$, Nico de Jong ${ }^{\mathrm{a}, \mathrm{b}}$ \\ ${ }^{a}$ Acoustical Wavefield Imaging, ImPhys, Delft University of Technology, Delft, the Netherlands \\ ${ }^{\mathrm{b}}$ Biomedical Engineering, Thorax Center, Erasmus MC, Rotterdam, the Netherlands \\ ${ }^{\mathrm{c}}$ Department of Information Engineering, University of Florence, Florence, Italy \\ ${ }^{\mathrm{d}}$ Lab. on Cardiovascular Imaging \& Dynamics, Dept. of Cardiovascular Sciences, KU Leuven, Leuven, Belgium \\ Corresponding author: H.Vos@ErasmusMC.nl
}

\begin{abstract}
Volumetric medical ultrasound imaging currently provides either high resolution or high frame rates. We propose a sparse array probe with only 256 elements that are density tapered. All elements are directly wired to an ultrasound engine, thus being flexible in transmission and reception. Here we present the design, realization, and characterization of a probe prototype for carotid 3D high frame rate scanning $(5 \mathrm{MHz})$. The design is based on rapid prototyping with printed circuit board substrates to wire the elements to external connectors. The results show that $95 \%$ of the elements was acoustically active, producing a peak pressure of $400 \mathrm{kPa}$ when focused at $24 \mathrm{~mm}$ with a $20 \mathrm{~V}$ amplitude, 3cycle tone burst. The fractional one-way $-6 \mathrm{~dB}$ bandwidth (FBW) was $32 \%$, which is narrower than the designed one $(50 \% \mathrm{FBW})$, due to an unexplained ringing effect. These results show that the probe is viable for volumetric imaging tests, albeit that it can be further optimized.
\end{abstract}

\section{Keywords-Ultrasound, sparse, spiral, probe, PZT-on-PCB}

\section{INTRODUCTION}

Volumetric medical ultrasound imaging currently provides either high resolution or high frame rates. High resolution is obtained by commercial clinical 3D systems, which reach volume rates of up to 25 Volume Frames Per Second (VFPS). High volumetric frame rates have been achieved by e.g. $[1,2]$. Efforts to obtain both at the same time involve either expensive and complex multi-channel $(1000+)$ ultrasound systems $[3,4]$ or in-probe electronics to reduce cable count [5].

Each element can be separately addressed in the large aperture, opposite to current trends in volumetric probes where the high element count is faced with sub-aperture beamforming, serial readouts, row-column addressed arrays, or limited aperture sizes [2, 5-7].

A trade-off between resolution, contrast, frame rate and imaging depth can be found in so-called sparse arrays, in which the acoustic elements only sparsely cover the overall probe aperture, instead of the full population of the probe area $[2,3,8,9]$. The sparsity reduces element count, thus relaxing the number in channel count from, roughly, 1000+ elements to 256. Several 256-channel systems are currently available which facilitates the use of such 256-element systems [4]. In sparse arrays, the elements are preferably located with random mutual spacing, in order to avoid grating lobes in transmit and receive, typically caused by uniform spacing. Moreover, density tapering has been earlier proposed to simultaneously reduce off-beam signal levels and maintain good signal-to-noise ratio, since the density tapering provides a natural aperture window function which would otherwise be achieved by amplitude-weighting of the signals in the aperture $[10,11]$.

The full control over transmit waveforms per elements allows several imaging strategies. One direction of possible imaging strategy is multi-beam transmits in which highly focused beams can be simultaneously steered to several
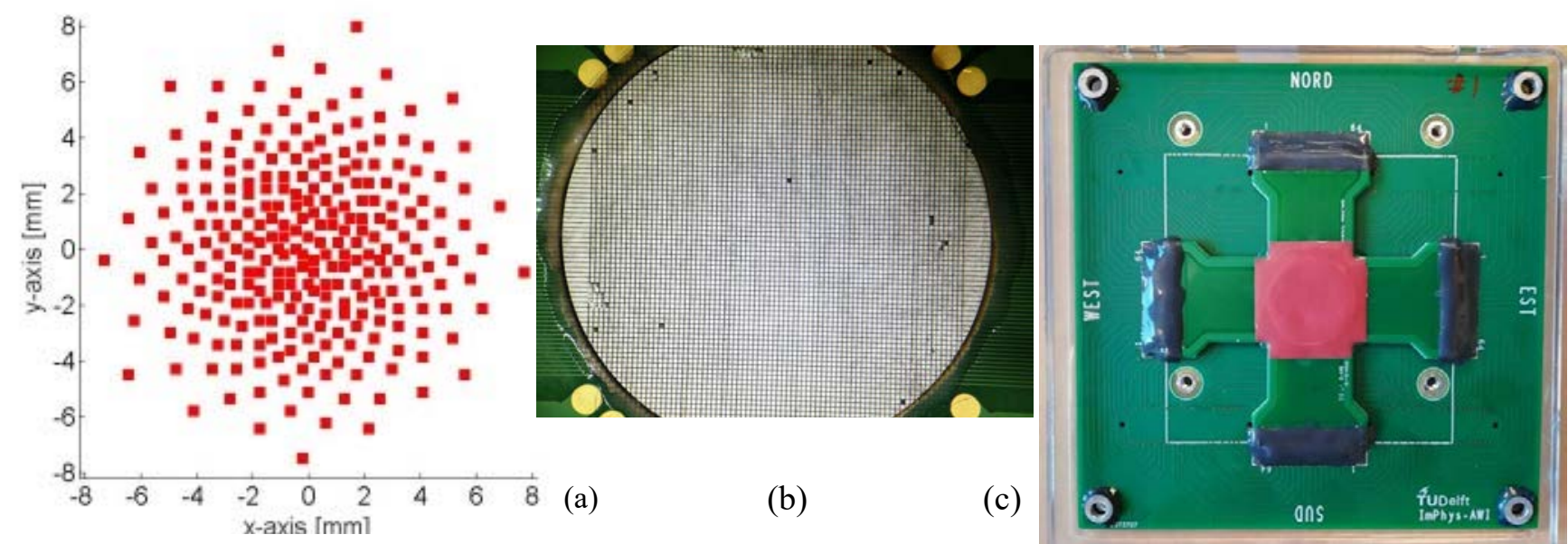

Fig. 1. (a) Element topology; (b) photograph of elements after cutting, before applying ground foil and protective layer; (c) photograph of probe built on PCB. The cross-shaped PCB is the basis of the probe; the active area is covered with pink protective silicone. The square PCB behind it facilitates the connection of this first prototype to the cables. 
volumetric directions and several image lines per transmit event are reconstructed $[12,13]$. This does require adequate waveform control as several distinct pulses must be fired within one transmit event. Compared to fully populated probes with sub-aperture beamforming or other forms of multiplexing, a sparse array is the only solution that enables high frame-rate, high-resolution multi-beam transmits and multi-line acquisition. One advantage of the beam pattern of multi-beam transmits is an overall reduction of contrast agent destruction, compared to that in the near field of diverging wave beams [14]. Motion artefacts are also minimal as no coherent compounding is used. Another direction could be diverging wave compounding [3], that has lower demands on the transmission electronic circuitry as only a single pulse per element per event is transmitted.

We propose a sparse array probe with 256 elements and a density-tapered topology, based on a printed circuit board prototyping procedure. All elements are directly wired to an ultrasound engine, thus being flexible in transmission and reception. Here we present the design, realization, and characterization of a probe prototype for carotid 3D high frame rate scanning.

\section{Materials AND MethodS}

\section{A. Design}

The distribution of the 256 elements was based on a density-tapered spiral [10] (Fig. 1a). The original element positions proposed by Ramalli et al. [10] were snapped onto a $220 \times 220 \mu \mathrm{m}$ grid to enable conventional transducer dicing in the production phase. At $5 \mathrm{MHz}$, a pitch of $220 \mu \mathrm{m}$ would result in grating-lobe-free steering up to $23^{\circ}$. The aperture diameter was $16 \mathrm{~mm}$ and element sizes were $200 \times 200 \mu \mathrm{m}$ after cutting.

We built the probe as PZT-on-PCB, partly expanding on our earlier PZT-on-ASIC technology that was described for a micro-TEE matrix transducer [15]. As shown in Fig. 1a, the element locations were converted to a PCB design in which all element pads were directly wired to connector locations on the ends of four arms, each connecting 64 channels with sufficient number of ground pins. This PCB design was realized in rigid high-precision rapid prototyping $\mathrm{PCB}$ technology (RushPCB, San Jose, CA), with a base thickness of $1.55 \mathrm{~mm}$, copper traces of $17 \mu \mathrm{m}$, and soft-gold finish facilitating good bonding of gold balls. Gold balls, one per element location, were applied on top of the PCB element pads. A non-conductive epoxy was deposited between the gold balls to form the substrate to the elements, and this layer was ground down to expose the gold balls, forming electrical contact points for the PZT stack. Besides functioning as electrical isolation, the non-conductive epoxy also acts as a mechanical dicing buffer (buffer layer) with a thickness of roughly $30 \mu \mathrm{m}$. Next, the acoustic stack was mounted which consisted of an electrically conductive epoxy glue (acoustic impedance of 4.7 MRayl) of $60 \mu \mathrm{m}$ thickness on either side of a $225 \mu \mathrm{m}$ thick high-density piezoceramic (3202HD, CTS Corp.). The electrically conductive glue on the back side provides the electrical connection between the PZT and the exposed gold balls. The regular element pattern was cut with conventional dicing saw (Fig. 1b). The conductive glue on the front side acts both as acoustic matching layer and as electrical connection to ground via a thin conductive aluminum foil. Finally, the aperture was covered with a thin $(<50 \mu \mathrm{m})$ layer of silicone rubber for protection of the
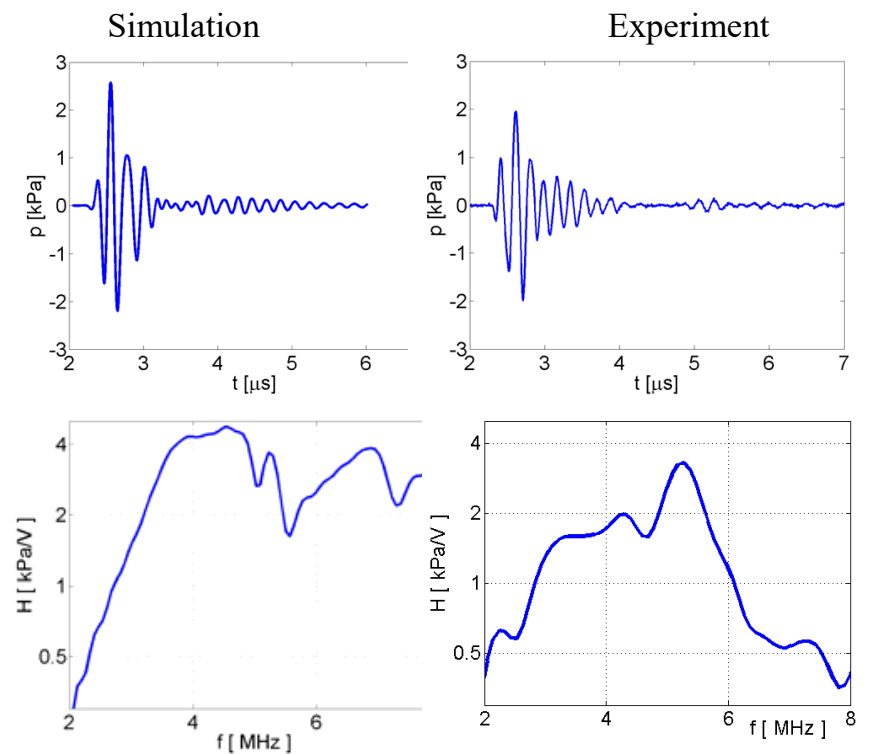

Fig. 2. Pressure pulses and transfer function (in measurement: average of 4 elements) of single elements, measured at a distance of $3.4 \mathrm{~mm}$ from the surface, in simulation and experiement.

conductive foil and mounted on a regular-process connection-PCB (Fig. 1c).

Receive signal amplification and line driving was performed with MAX4805 high-voltage-protected OpAmps (MAXIM Integrated, San Jose, CA) mounted on custom designed PCBs. Special care was taken on-board to reduce noise on the power supply and high-voltage (substrate) lines of the MAXIM OpAmps, as such noise would lead to severe common-mode noise in the amplified signals.

\section{B. Simulation}

A simulation in finite-difference numerical software (PZFlex release 4.0, Weidlinger Associates Inc., Mountain View, CA) was prepared to predict the acoustic behavior of isolated elements. The relevant PCB material properties were found by additional acoustic measurements as $\mathrm{c}=3153 \mathrm{~m} / \mathrm{s}, \rho$ $=1938 \mathrm{~kg} / \mathrm{m}^{3}, \mathrm{Z}=6.1$ MRayls, which match reasonably well with the properties found for FR4 material in the software package( $\mathrm{c}=3369 \mathrm{~m} / \mathrm{s}, 1850 \mathrm{~kg} / \mathrm{m}^{3}, \mathrm{Z}=6.2$ MRayls). The PCB bottom side had a reflective boundary condition in the simulation.

The predicted transmission pulse and its spectrum are displayed in Fig. 2-left, showing a center frequency of $4.5 \mathrm{MHz}$, and several higher-frequency resonance modes due to lateral resonance and the multi-layer structure of the design. 


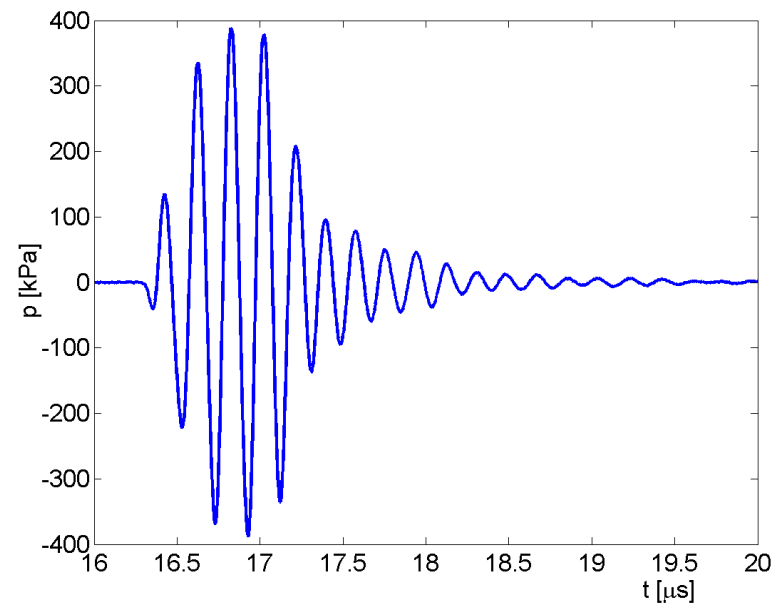

Fig. 3. Acoustic pulse when full probe is focused at $24 \mathrm{~mm}$.

\section{Characterization}

The probe was connected to a Vantage-256 scanner (Verasonics, Kirkland WA) for acoustic characterization of single elements and of the full probe in a water bath. In all separate transmit and receive measurements, the probe was mounted co-axially on a rotational axis, whereas the opposite transducer was mounted on a XYZ translation stage. This facilitated full alignment. Transmitted pulses were recorded with a calibrated 200- $\mu \mathrm{m}$ PVDF needle hydrophone (Precision Acoustics, Dorchester, UK). Receive sensitivity was measured by insonification with a calibrated $5-\mathrm{MHz}$ unfocused transducer (V309, 0.5-inch diameter, Olympus Scientific Solutions, Waltham, MA, USA) in far field $(30 \mathrm{~cm})$ [15]. Beam profiles were recorded with angular steps of $0.5^{\circ}$.

Element variability was measured in a pulse-echo setup, in which a steel reflector was located at a distance of $6.7 \mathrm{~mm}$ from the probe, in which the gap was filled with acoustic gel. All elements were probed sequentially in transmit-receive mode with the research scanner with a 3-cycle wave of $5 \mathrm{MHz}$.

\section{RESULTS AND DISCUSSION}

The transmit efficiency of a single element was $3.5 \mathrm{kPa} / \mathrm{V}$ at $3.4 \mathrm{~mm}$ distance, see Fig. 2-right for the measured pulse and the transfer function (average of four elements, compensated for the electric driving signal). Driving signal was a 1.5 cycle Rickert wavelet. The center frequency was $5.5 \mathrm{MHz}$, and fractional one-way $-6 \mathrm{~dB}$ bandwidth (FBW) was $32 \%$, narrower than the designed one $(50 \% \mathrm{FBW})$. We also noticed a ringing effect in the measured pulse that was most likely caused by the absence of an absorbing layer behind the PCB and hence reverberation in the $\mathrm{PCB}$ material, albeit the simulated element had similar absence of absorbing material behind the PCB.

The sensitivity measurements (data not shown) showed that $95 \%$ of the elements was acoustically active, and $87 \%$ was within $\pm 3 \mathrm{~dB}$ signal amplitude range compared to the average of the active elements, in pulse-echo.

When the full probe was focused at $24 \mathrm{~mm}$ with a $20 \mathrm{~V}$ amplitude, 3-cycle tone burst, the peak pressure in focus was $400 \mathrm{kPa}$ (Fig. 3). Such focal pressure at $5 \mathrm{MHz}$ is generally not sufficient to generate nonlinear propagation, but it is sufficient for a penetration depth of few $\mathrm{cm}$ in fundamental imaging, as needed in carotid imaging.

Fig. 4 shows several examples of steered beams in a range $\left[-45^{\circ},+45^{\circ}\right]$. As expected from simulations $[11,13]$ the beams have no apparent sidelobes, albeit that the transmission 'clutter' is relatively high $(-23 \mathrm{~dB})$ compared to fully populated arrays (lower than $-40 \mathrm{~dB}$ ). The one-way two-sided $-6 \mathrm{~dB}$ opening angle of the probe was $80^{\circ}$ (trend curve in Fig. 4) and, consequently, so was the average of the individual elements. This is smaller than the opening angle of a piston source with a typical size of $200 \mu \mathrm{m}\left(120^{\circ}\right.$ at 5 $\mathrm{MHz}$ in tissue) but sufficient to capture a large field of view on the carotid artery.

The OpAmps improved SNR by $8 \mathrm{~dB}$ with $1 \mathrm{~m}$ of coaxial cable attached. We expect to improve SNR further by proper electric shielding of the currently bare electric signal traces between the active elements and connectors which thus reduces the common mode noise.

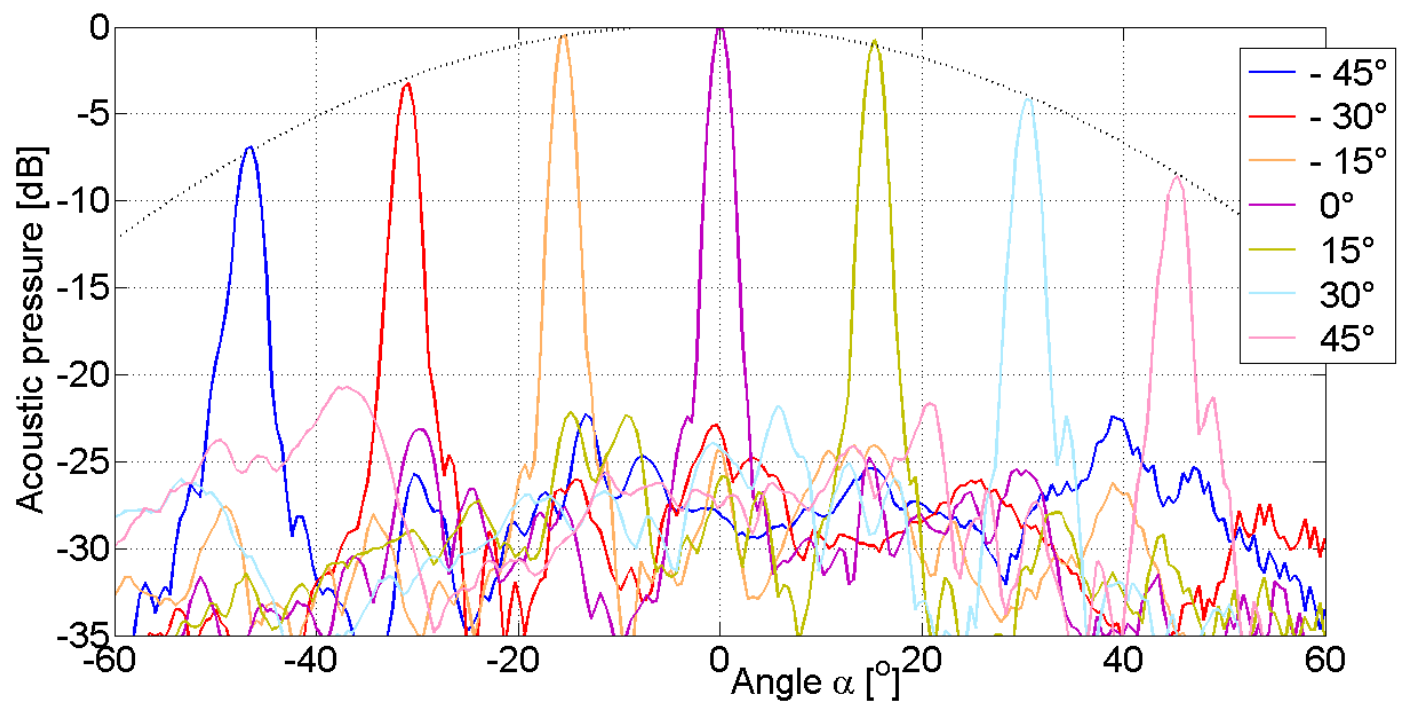

Fig. 4. Beamprofiles when steering to angles within the range of -45 to 45 degrees. 
In preliminary functional tests, we could detect invisibly small air bubbles in tap water in pulse-echo mode with a single diverging transmit wave when connected to the Verasonics system. Multi-line transmits were generated by the probe when driven by an experimental imaging system containing 256 arbitrary waveform generators [16].

\section{CONCLUSION}

Although the bandwidth and length of the transmitted pulse needs further improvements, the measurements show that a sparse array can be built on rapid prototyping printed circuit boards with high fidelity. The results show that the probe is viable for at least in-vitro tests and can thus act as a platform for testing volumetric imaging sequences based on sparse arrays.

\section{ACKNOWLEDGMENT}

We thank Henry den Bok of TU Delft for his help in developing the PCB.

\section{REFERENCES}

[1] J. Sauvage, M. Flesch, G. Ferin, A. Nguyen-Dinh, J. Poree, M. Tanter, M. Pernot, and T. Deffieux, "A large aperture row column addressed probe for in vivo 4D ultrafast doppler ultrasound imaging," Physics in Medicine \& Biology, in press, DOI https://doi.org/10.1088/1361-6560/aae427, 2018.

[2] J. Provost, C. Papadacci, J. E. Arango, M. Imbault, M. Fink, J.-L. Gennisson, M. Tanter, and M. Pernot, "3D ultrafast ultrasound imaging in vivo," Physics in Medicine \& Biology, vol. 59, p. L1, 2014.

[3] E. Roux, F. Varray, L. Petrusca, C. Cachard, P. Tortoli, and H. Liebgott, "Experimental 3-D Ultrasound Imaging with 2-D Sparse Arrays using Focused and Diverging Waves," Scientific Reports, vol. 8, p. 9108, 2018.

[4] E. Boni, A. Yu, S. Freear, J. A. Jensen, and P. Tortoli, "Ultrasound Open Platforms for Next-Generation Imaging Technique Development," IEEE Transactions on Ultrasonics, Ferroelectrics, and Frequency Control, vol. 65, pp. 1078-1092, 2018.

[5] C. Chen, Z. Chen, D. Bera, S. B. Raghunathan, M. Shabanimotlagh, E. Noothout, Z. Y. Chang, J. Ponte, C. Prins, H. J. Vos, J. G. Bosch, M. D. Verweij, N. De Jong, and M. A. P. Pertijs, "A Front-End ASIC with Receive Sub-array Beamforming Integrated with a $32 \times 32$ PZT Matrix Transducer for 3-D Transesophageal Echocardiography," IEEE Journal of Solid-State Circuits, vol. 52, pp. 994-1006, 2017.

[6] P. Santos, G. U. Haugen, L. Løvstakken, E. Samset, and J. D'hooge, "Diverging Wave Volumetric Imaging Using Subaperture
Beamforming," IEEE Transactions on Ultrasonics, Ferroelectrics, and Frequency Control, vol. 63, pp. 2114-2124, 2016.

[7] T. L. Christiansen, M. F. Rasmussen, J. P. Bagge, L. N. Moesner, J. A. Jensen, and E. V. Thomsen, "3-D imaging using row-columnaddressed arrays with integrated apodization - part ii: Transducer fabrication and experimental results," IEEE Transactions on Ultrasonics, Ferroelectrics, and Frequency Control, vol. 62, pp. 959971, 2015.

[8] A. Austeng and S. Holm, "Sparse 2-D arrays for 3-D phased array imaging - design methods," IEEE Transactions on Ultrasonics, Ferroelectrics, and Frequency Control, vol. 49, pp. 1073-1086, 2002.

[9] B. Diarra, H. Liebgott, M. Robini, P. Tortoli, and C. Cachard, "Novel strategies in 2D sparse arrays for 3D ultrasound imaging," Physica Medica: European Journal of Medical Physics, vol. 32, pp. 420-421, 2016.

[10] A. Ramalli, E. Boni, A. S. Savoia, and P. Tortoli, "Density-tapered spiral arrays for ultrasound 3-D imaging," IEEE Transactions on Ultrasonics, Ferroelectrics, and Frequency Control, vol. 62, pp. 1580$1588,2015$.

[11] E. Roux, A. Ramalli, P. Tortoli, C. Cachard, M. C. Robini, and H. Liebgott, "2-D ultrasound sparse arrays multidepth radiation optimization using simulated annealing and spiral-array inspired energy functions," IEEE Transactions on Ultrasonics, Ferroelectrics, and Frequency Control, vol. 63, pp. 2138-2149, 2016.

[12] A. Ortega, J. Provost, L. Tong, P. Santos, B. Heyde, M. Pernot, and J. D'hooge, "A Comparison of the Performance of Different Multiline Transmit Setups for Fast Volumetric Cardiac Ultrasound," IEEE Transactions on Ultrasonics, Ferroelectrics, and Frequency Control, vol. 63, pp. 2082-2091, 2016

[13] A. Ramalli, P. Santos, P. Tortoli, and J. D'hooge, "Tri-plane cardiac imaging using multi-line transmission on a spiral array: a feasibility study," in IEEE Int. Ultrasonics Symposium, Kobe, Japan, 2018.

[14] M. Toulemonde, Y. Li, S. Lin, F. Cordonnier, M. Butler, W. C. Duncan, R. J. Eckersley, V. Sboros, and M. X. Tang, "High-FrameRate Contrast Echocardiography using diverging waves: initial invitro and in-vivo evaluation," IEEE Transactions on Ultrasonics, Ferroelectrics, and Frequency Control, in press, DOI 10.1109/TUFFC.2018.2856756, 2018.

[15] V. Daeichin, D. Bera, S. Raghunathan, M. Shabani Motlagh, Z. Chen, C. Chen, E. Noothout, H. J. Vos, M. Pertijs, J. G. Bosch, N. de Jong, and M. Verweij, "Acoustic characterization of a miniature matrix transducer for pediatric 3D transesophageal echocardiography," Ultrasound Med Biol, vol. 44, pp. 2143-2154, 2018.

[16] E. Boni, A. Ramalli, V. Daeichin, N. De Jong, H. J. Vos, and P. Tortoli, "Prototype 3D real-time imaging system based on a sparse PZT spiral array," in IEEE Int. Ultrasonics Symposium, Kobe, Japan, 2018 . 